\title{
Der ungarische Lebensbezirk im literarischen Schaffen von Emil Witting Ausgehend von einer neueren Veröffentlichung
}

\section{Joachim WITTSTOCK}

Dr.h.c., Schriftsteller und Literaturwissenschaftler, Sibiu/Hermannstadt E-Mail: wittstock.joachim@gmail.com

\begin{abstract}
The writer Emil Witting (1880-1952), known by German readers through the descriptions of the forests and pastures of the Carpathian Mountains, author of extensive relations dedicated to the bear (Frate Nicolae) and to deer (Scrimerul), conceives a novel dedicated to a painter connected to the Szekler's world. Imre Nagy (1893-1976) served as a model for the main character. From this unfinished writing, three fragments were published. These have recently been translated into Hungarian, printed in Miercurea Ciuc in an illustrated edition containing Imre Nagy's paintings and graphic works.
\end{abstract}

Key words: German literature of Romania in the inter-war period; the writer Emil Witting; the Hungarian sphere of existence in Transylvania as a literary theme; the Szekler painter Imre Nagy

Ohne Impulse von der ungarisch-szeklerischen Seite unserer siebenbürgischen Welt wäre ich nicht veranlasst gewesen, mich über Emil Witting (1880-1952) zu äußern. Die Anstöße aus Szeklerburg, will sagen aus Miercurea Ciuc beziehungsweise Csíkszereda, regten mich und meinen Sinn für eine gewisse Vollständigkeit dazu an, nicht nur die im Vordergrund stehenden ungarischen Beziehungen im Schaffen des Schriftstellers zu beleuchten, sondern ein Streiflicht auch auf seine Wahrnehmung des rumänischen Volkslebens zu lenken und auch die Roma nicht zu übergehen. 
Witting selbst vertrat ja die Meinung, die so ganz in die heutigen Vorstellungen vom Zusammenleben in einer Region passt: „Erst wenn wir ganz das Ursein unserer Landschaft, die uns umgebenden Völker kennen und verstehen, werden wir uns selbst begreifen können, sind wir doch unter ihnen, mit ihnen zu dem geworden, was wir sind. “1

(In Klammer: Wir wollen hier nicht der Versuchung nachgeben, den Kontext zu beschreiben, in dem Witting 1934 sich für die bodenständige, regional gebundene Literatur Siebenbürgens einsetzte, weil unsere Ausführungen eine bestimmte Richtung einzuschlagen und zu halten haben. Nur soviel sei hier angemerkt: Witting vertrat damals die Auffassung, siebenbürgisch-sächsische Schriftsteller dürften sich nicht - wegen größerer Auflagen und damit höherem Ansehen - zu sehr auf reichsdeutsche Publikationsmöglichkeiten und damit auf die Erwartungen eines binnendeutschen Publikums einstellen; „unser Hauptanker" habe vielmehr „das Wesentliche in der Seele der uns umgebenden Völker, unseres Volkes selbst" zu sein. $^{2}$ )

Die Antriebe, mich an Emil Witting, an seine Biographie und Schriftstellerei zu erinnern und mein Wissen davon - zu meinem Nutzen - auch zu bereichern, hängt mit der Entstehung eines Buches zusammen, von dem ich, als Betrachter aus der Ferne, Kenntnis erhalten hatte. Als es erschienen war, bin ich von seiner Komplexität überrascht gewesen, nahm ich doch wahr, es habe zwar Emil Wittings Hinwendung zum Ungarntum zum Ausgangspunkt, biete jedoch mehr. Und das durch bemühtes Erkunden der Hintergründe von Wittings diesbezüglichen Erfahrungen, durch kundiges Veranschaulichen dessen, was anhand zahlreicher Details vor allem aus dem bildkünstlerischen Bereich über den Gesichtskreis des siebenbürgisch-sächsischen Schriftstellers von dazumal hinausging.

1 Witting, Emil: Aufgaben des siebenbürgisch-sächsischen Schrifttums. In: Klingsor, 11. Jg., Heft 3, März 1934, S. 122.

2 Ebenda, S. 121. 
Der Titel der gemeinten Veröffentlichung ist deutsch, Der Maler Nagy Imre, ansonsten aber ist alles Weitere magyarisch abgefasst, selbst der Untertitel, A székely festö és az erdélyi szászok (1925-1935). Emil Witting regényrészleteivel. Übersetzt lautet dieser: Der Szekler Maler und die Siebenbürger Sachsen (1925-1935). Emil Wittings Romanfragmente. Die Autoren des Bandes sind Béla János Bács - zuständig für die Übersetzung und Deutung der Wittingschen Texte - und Róbert Kálmán Ráduly, der den bildkünstlerischen Teil beisteuerte. Erschienen ist das Buch 2018 im Verlag des Bürgermeisteramtes von Miercurea Ciuc / Csíkszereda / Szeklerburg. ${ }^{3}$

Allzu geringe Sprachkenntnisse hindern mich daran, auf das spezifisch ungarische Moment des Textes einzugehen, ich bin vielmehr darauf verwiesen, die dem Buch zugrunde liegenden deutschen Quellen zu kommentieren. Da sind vor allem drei Fragmente aus einem Romanprojekt, dessen Hauptgestalt ungarischer Volkszugehörigkeit ist.

Wie kam es zu jener Romankomposition? Wer war ihr Autor Emil Witting, wer der Protagonist?

Das Buch Die rumäniendeutsche Literatur in den Jahren 1918-1944, schon in den letzten Siebzigerjahren konzipiert und in den Achtzigerjahre ausgeführt, aber erst nach der politischen Wende erschienen (1992), stützte sich im Kapitel Emil Witting auf die in Hermannstadt erreichbaren Daten. Der Autor des Kapitels, unser einstiger Kollege Udo Peter Wagner ${ }^{4}$, konnte bei der Dokumentation feststellen, dass sich hierorts Biographie

3 Bács, Béla János/Ráduly, Róbert Kálmán: Der Maler Nagy Imre. A székely festö és az erdélyi szászok (1925-1935). Emil Witting regényrészleteivel. Csíkszereda: Csíkszereda Kiadóhivatal 2018 [Der Maler Nagy Imre. Der Szekler Maler und die Siebenbürger Sachsen (19251935). Emil Wittings Romanfragmente. Miercurea Ciuc/Szeklerburg: Verlag des Bürgermeisteramtes]. Mit zahlreichen Reproduktionen von Graphiken und Ölbildern des Künstlers.

4 Wagner, Udo Peter: Emil Witting. In: Die rumäniendeutsche Literatur in den Jahren 1918-1944. Redigiert von Wittstock, Joachim/Sienerth, 
und Schaffen des Schriftstellers ganz gut erfassen ließen. Er hat nicht nur Druckschriften aus öffentlichen und privaten Bibliotheken durchgesehen, sondern auch noch einen - umfangmäßig verhältnismäßig bescheidenen - Nachlassbestand gesichtet (einst bei Familie Petzel, ein heute nicht mehr zu eruierendes Dokumentationsmaterial).

Eine kurzgefasste Biographie von Emil Witting beläuft sich auf hier und dort vermerkte Daten: Geburt in Kronstadt/Braşov 1880, Besuch des Honterusgymnasiums daselbst, Studium der Forstwissenschaft in Schemnitz, ungarisch Selmecbánya, slowakisch (weil in der heutigen Slowakei gelegen) Banská Štiavnica. Witting war Forstadjunkt in Kronstadt, 1908 übersiedelte er nach Hermannstadt/Sibiu. Er war Oberförster und leitender Forstmeister der im Südwesten Hermannstadts gelegenen Siebenrichterwaldungen, bis 1928. Hierauf als Verfasser von Tierschilderungen, Naturbeschreibungen und Jagderlebnissen tätig; er war Redakteur der Zeitschrift KarpathenWaidwerk (1929 und 1930 in Hermannstadt erschienen) und Mitarbeiter verschiedener heimischer Publikationen.

Bekannt wurde er vor allem durch seine Romane: Frate Nicolae, der siebenbürgische Karpathenbär. Eine Lebensgeschichte, erst im Selbstverlag des Verfassers publiziert, Hermannstadt 1931, dann auch vom Verlag Rütten \& Loening in Potsdam 1939 herausgebracht.

WeiterhingestalteteerEinLebensbilddes Karpathenhirsches und benannte es Der Fechter. Auch dieses Buch erschien erst im Selbstverlag, 1935 und wurde später von Rütten \& Loening (1937) übernommen und mit Fotografien ausgestattet. Das Werk ist nach dem Zweiten Weltkrieg vom Grazer LeykamVerlag erneut ans naturverbundene Lesepublikum herangetragen worden (1951), versehen mit Zeichnungen des siebenbürgischen Graphikers Fritz Kimm. Von den Tierromanen gab es noch weitere Ausgaben.

Stefan. Bukarest: Kriterion Verlag 1992 (Beiträge zur Geschichte der rumäniendeutschen Dichtung), S. 217-225, 510f, Bildteil S. 14f. 
Beachtung fand auch Hirtenfeuer. Ein Karpathenroman, 1932 im Hermannstädter Krafft \& Drotleff Verlag erschienen, als Band 2 der Deutschen Buchgilde in Rumänien. Ein Jahreslauf in der Bergwelt wird geschildert, eine Szenerie mit dörflicher Siedlung, vor allem aber mit Waldlandschaft und Alm, mit den Sennereien der rumänischen Hirten. Es ist ein Universum für sich, voller Herbheit, urtümlicher Kraft und verborgenem oder auch sichtbarem, den Betrachter gar überwältigenden Reiz.

Die „Würdigung des Volkstums der Rumänen“, schrieb Alfred Hönig, der Zeitungsredakteur und gelegentliche Jagdgefährte des Schriftstellers, „,brachte Wittings Büchern viel Beachtung und Lob auch bei rumänischen Jägern und Lesern

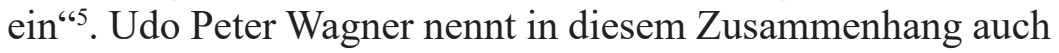
Mihail Sadoveanu, den Witting ,bei der Jagd kennengelernt hatte und mit dem er auch im Briefwechsel stand", durch dessen Vermittlung Witting zudem „eine Pension von seiten des Schriftstellerverbandes" " erhielt. $^{6}$

Witting lässt Wido Walding, sein Alter ego, die Abläufe in der Natur beobachten und erleben und führt auch das Menschenwerk in guten und weniger guten Taten vor, indem er redliche Hauswirte, tüchtige Unternehmer der Weidewirtschaft, aber auch zwielichtige Geschäftsleute, Schmuggler und Wilderer auftreten lässt.

Das Besondere von Landschaft, Tier und Mensch anzudeuten oder eindringlich zu kennzeichnen, ist der Autor immerfort bemüht, wobei er eine höchst eigenwillige Sprache verwendet, voll urwüchsiger Fügungen und über das Alltagsdeutsch hinausführenden Bezeichnungen aus Pflanzen- und Tierkunde, aus dem Jägeridiom, zudem aus der Überlieferung und Redeweise des rumänischen Volkes.

5 Hönig, Alfred: Emil Witting. Der Karpatenjäger, der zum Dichter wurde. In: Südostdeutsche Vierteljahresblätter, 29. Jg., 1980, Folge 4, S. 274.

6 Wagner, Udo Peter: Emil Witting, a. a. O., S. 218. 
Die stilistischen Eigenheiten Wittings kennzeichnete Alfred Hönig mit den Worten:

Die Sprache, in der Wittings Werke geschrieben sind, ist reich an Neuschöpfungen und Wiederentdeckungen untergegangenen Sprachgutes; ähnlich wie bei Hermann Löns, dessen Einfluss überhaupt oft erkennbar ist. Es ist eine knorrige, oft wie Wurzelwerk einer alten Schirmfichte verästelte Satzbildung, umstritten wegen ihrer manchmal ungefügen sperrigen Verflechtung, wenn sie sich in Unwägbares, Rätselhaftes und Undeutbares verstrickt. Und doch erhebt sie sich immer wieder zu starker Leuchtkraft... ${ }^{7}$

Nach Aufenthalten in verschiedenen Quartieren wohnte Emil Witting in der von ihm errichteten Villa, zusammen mit der Gattin Maria geb. Schuster und den drei Kindern, die Lili Kriemhild, Siegfried Friedrich und Dietlind benannt worden waren. Heute heißt die Straße ihres Zuhauses Strada Cristian, früher war sie für gewisse Zeitabschnitte mit den Namen Vasile Goldiş, Ioan Barac und Alexandrov belegt. Der unweit davon lebende Professor Volker Hermann bestätigte mir, welches Gebäude den Wittings gehört hatte (Nr. 9).

1952 ist Emil Witting in Hermannstadt gestorben, er wurde auf dem Zentralfriedhof bestattet. Sein Grabstein ist auch heute noch zu sehen.

$\mathrm{Zu}$ meiner Überraschung stellte ich vor nicht allzu langer Zeit im Internet fest, Wittings Nachkommen hätten einen in Deutschland befindlichen Nachlass des Schriftstellers dem Münchner Südostdeutschen Kulturwerk übergeben und damit indirekt die Nachfolgeeinrichtung beliefert, das Institut für deutsche Kultur und Geschichte Südosteuropas (IKGS). Ein „Findbuch“ zu dieser literarischen Hinterlassenschaft liegt vor. ${ }^{8}$

Aus ihm ist ersichtlich, dass der Roman, der uns zu beschäftigen hat, dort vorliegt; das Typoskript umfasst 309 „lose

7 Hönig, Alfred: Emil Witting, a. a. O., S. 274.

8 Archiv des IKGS. Findbuch zum Bestand Emil Witting (1880-1952).

Bearbeitet von Dr. Kramer, Rainer. München 2015, 17 S. 
Blätter, paginiert“. Titel und Widmung: „Maler der Heimat. Ein Szeklerroman. Dem Maler Nagy Imre.“ Keine Angaben zur Entstehung: Ohne Datum, ohne Ortsangabe.

Hierzulande zugänglich waren und sind drei Bruchstücke des Romans, in drei Veröffentlichungen einheimischer Presse einsehbar:

1. Pfingstwallfahrt. Ein Abschnitt aus dem Roman „Maler seines Volkes" (so der ursprüngliche Titel, später Maler der Heimat);

2. Im Zigeunerlager. Aus einem unveröffentlichten Roman ${ }^{10}$;

3. Letzte Fahrt. Aus einem unveröffentlichten Roman ${ }^{11}$.

1) Geschildert wird eine katholische Wallfahrt, die zu Pfingsten der Heiligen Maria gilt, mit Kirchgang und anschließender Prozession einen Berg hinan, mit geistlichen Handlungen im Bergsattel. Nach dem Abstieg entfaltet sich ein volksfestartiges Getriebe. Zahllose Menschen sind unterwegs, aus allen Gegenden gekommen, ,wo ungarische Zunge züngelte“"12, unter ihnen der junge Maler Géza. Er findet manches befremdlich an dem vielgestaltigen Geschehen, ist zudem ,ein Ungläubiger"13, wird dann aber doch von dem Ablauf der Festlichkeit gefesselt, er ,konnte sich der berauschenden Wirkung, der Suggestion des Zeremoniells kaum erwehren." ${ }^{14}$ Und das, obwohl er sich vorhält, da stehe „Aufmachung gegen Wirklichkeit, Idee gegen Realität", da lebe

9 Witting, Emil: Pfingstwallfahrt. In: Klingsor, 11. Jg., Heft 9, September 1934, S. 330-340.

${ }^{10}$ Witting, Emil: Im Zigeunerlager. In: Klingsor, 12. Jg., Heft 6, Juni 1935, S. 218-224.

11 Witting, Emil: Letzte Fahrt. In: Kronstädter Zeitung. Festausgabe zum hundertjährigen Bestehen. Sonntag, den 24. Mai 1936, S. 37-38.

Nachdruck im Verlag Neue Kronstädter Zeitung, München 1990.

${ }^{12}$ Witting, Emil: Pfingstwallfahrt, a. a. O., S. 331.

${ }^{13}$ Ebenda, S. 332.

${ }^{14}$ Ebenda, S. 334. 
„Formenschwulst und Gewoge“ sich aus, da herrsche „das Pastose der Farben“, zudem ,die Macht der Süßigkeit der Töne". ${ }^{15}$ Zwei ihm bekannte Frauen glaubt Géza im Gewimmel zu erkennen und überlegt sein Verhältnis zur einen, die für ihn der Vergangenheit angehört, und zur anderen, zu der er sich hingezogen fühlt, obwohl sie, die er einst gemalt hat, die Gattin eines anderen ist.

Der Ort, an dem die ungewöhnliche Veranstaltung abläuft, wird nicht genannt, doch denkt man unwillkürlich an die seit Jahrhunderten zu Pfingsten stattfindende Prozession in Şumuleu Ciuc/ Csíksomlyó, in einem OrtmitKircheund Kloster der Franziskaner unweit von Szeklerburg / Miercurea Ciuc / Csíkszereda (heute ist die Ortschaft verwaltungsmäßig der Stadt Miercurea Ciuc eingegliedert).

2) Vom Zigeunerlager verspricht sich der Maler Géza künstlerische Anregung und insgesamt die Bereicherung seiner Erfahrung. Um das Vertrauen der Halbnomaden zu gewinnen, nähert er sich in abgerissenen Kleidern der improvisierten Niederlassung, auch ist er bemüht, sich dem da herrschenden zwanglosen Verhalten anzupassen. Das gelingt ihm auch, wenngleich ein gewisses Misstrauen bleibt, namentlich auch bei dem Anführer der Gruppe, dem Vătaf.

Gézas Absicht ist es, nicht nur einzelne Skizzen zu zeichnen, sondern auch ein Porträt der ihn sinnlich reizenden Dianetta zu malen, einer jungen Angehörigen des Stammes, die ihm bereits flüchtig bekannt war. $\mathrm{Zu}$ gründlicherem Schaffen ist bei dem unruhigen Getriebe ringsum nicht recht Gelegenheit, auch wird Géza noch zu sehr als Eindringling gesehen. Er selbst hat anfängliche Hemmungen zu überwinden, er gibt sich freier als sonst und gliedert sich gar in einen spontan einsetzenden Tanz aller ein, in ihre rauschhafte Ekstase.

${ }^{15}$ Ebenda, S. 334. 
Das Romankapitel kann man zu besserem Verständnis im Zusammenhang mit einem Aufsatz lesen, den Emil Witting Über unsere Zigeuner geschrieben und im Klingsor veröffentlicht hatte (1935). In den seither verstrichenen acht Jahrzehnten hat sich im Dasein dieser Ethnie vieles verändert, und so darf man Wittings Ausführungen über die einzelnen Gruppen und Schichten der „Romi“, über ihre Lebensweise, Sprache, Überlieferung und ihre Zukunftsperspektiven als historisches Dokument der Landeskunde werten.

Bezeichnend ist die Stellung, die Witting dem unsteten Volk gegenüber einnimmt. Seine Anteilnahme an dem ,Zigeunervölkchen der lockeren Ansiedlung"ergibt sich aus der Erkenntnis, dass

auch dies arme Gesippe seinen Platz im mahlenden und zermalmenden Mühlengetriebe des Menschwerdens und Sterbens ebenso gesetzmäßig und rechtmäßig einnimmt wie jedes noch so hochgestellte Volk, das über seinem Hochflug hochmütig vergisst, welch Handlangern es seine Überlegenheit verdankt. ${ }^{16}$

Andererseits ist Witting zurückhaltend, wenn er daran denkt, dass der Zigeuner seine Freiheit und auch Sprache aufgeben wird und ,in die Welt der Ordnung und Haltung ${ }^{617}$ einzieht.

3) Der Maler Géza (nunmehr auch mit Familiennamen versehen, der freilich gleichzeitig Gattungsbezeichnung ist: Székely / der Szekler) - der Maler Géza also erscheint im Romanfragment Letzte Fahrt in einer neuen Eigenschaft, nämlich als Unternehmer im Bereich der Waldwirtschaft. Er heuert Arbeiter an, die zum Floß verbundene Baumstämme vom Oberlauf des Mieresch bis nach Sächsisch Regen / Reghin befördern, auf einem wegen Felsenge und bisweilen Hochflut gefährlichen Wasserweg. Einem

${ }^{16}$ Witting, Emil: Über unsere Zigeuner. In: Klingsor, 12. Jg., Heft 3, März 1935, S. 102.

${ }^{17}$ Ebenda, S. 107. 
erfahrenen Flößer, Ávéd Mózes, und dessen Sohn schließt Géza sich auf einer abenteuerlichen Fahrt an, trotz ungünstiger Witterung. Mit Mühe gelingt es Géza, sich aus den unversehens angewachsenen Fluten, aus den Strudeln der Felsklamm zu retten und auch den jungen Mann auf die Floßtafel zu ziehen - der dritte aber, der alte Ávéd Mózes, fehlt.

Braun und schwer schwamm das Wasser in dem kalten triefenden Nebelschauer auseinander, die Wellen platschten schwellend über Bord, nun fielen sie zusehends ab, das Floß schien gehoben, stellte sich langsam in die Fahrt und trieb still trauernd der Ungewissheit $\mathrm{zu}^{18}$

Witting hat - das lesen wir bei Udo Peter Wagner - im jungen Maler Géza ,einen Wahlverwandten“ gesehen, gezeichnet nach dem Prototyp Nagy Imre. ${ }^{19}$

Dieser authentische Heimatmaler wurde 1893 in JigodinBăi / Zsögödfürdö geboren, und lebte in der Dorfgemeinde nahe Miercurea Ciuc in einem Bauernhaus. Er starb 1976 in Miercurea Ciuc / Csíkszereda. Das ländliche Leben in seinem Geburtsort und in benachbarten Ortschaften war bevorzugtes Thema seiner Malerei. Wir dürfen mit dem Hinweis schließen, dass Emil Witting diesem Maler seines Volkes, Maler der Heimat, auch ein von Sympathie getragenes Essay gewidmet hat. $^{20}$

${ }^{18}$ Witting, Emil: Letzte Fahrt, a. a. O., S. 38.

19 Wagner, Udo Peter: Emil Witting, a. a. O., S. 217.

${ }^{20}$ Witting, Emil: Der Maler Nagy Imre. In: Klingsor, 11. Jg., Heft 4, April 1934, S. 129-133. 


\section{Literaturverzeichnis}

\section{Primärliteratur}

Witting, Emil: Frate Nicolae, der siebenbürgische Karpathenbär. Eine Lebensgeschichte. Hermannstadt/Sibiu: Selbst ${ }^{-}$ verlag des Verfassers 1931; Potsdam: Rütten \& Loening Verlag 1939.

Witting, Emil: Hirtenfeuer. Ein Karpathenroman. Hermannstadt: Verlag Krafft \& Drotleff 1932 (2. Band der Deutschen Buchgilde in Rumänien).

Witting, Emil: Der Fechter. Ein Lebensbild des Karpathenhirsches. Hermannstadt/Sibiu: Selbstverlag des Verfassers 1935; Potsdam: Rütten \& LoeningVerlag 1937. Mit 8 Bildtafeln; Graz: Leykam-Verlag 1951. Mit Zeichnungen von Fritz Kimm.

Witting, Emil: Aufgaben des siebenbürgisch-sächsischen Schrifttums. In: Klingsor, 11. Jg., Heft 3, März 1934, S. 119-122.

Witting, Emil: Der Maler Nagy Imre. In: Klingsor, 11. Jg., Heft 4, April 1934, S. 129-133.

Witting,Emil: Pfingstwallfahrt. In: Klingsor, 11. Jg., Heft 9, September 1934, S. 330-340.

Witting, Emil: Über unsere Zigeuner. In: Klingsor, 12. Jg., Heft 3, März 1935, S. 98-107.

Witting, Emil: Im Zigeunerlager. In: Klingsor, 12. Jg., Heft 6, Juni 1935, S. 218-224.

Witting, Emil: Letzte Fahrt. In: Kronstädter Zeitung. Festausgabe zum hundertjährigen Bestehen. Sonntag, den 24. Mai 1936, S. 37-38. Unveränderter Nachdruck im Verlag Neue Kronstädter Zeitung, München 1990.

\section{Sekundärliteratur}

Bács, Béla János / Ráduly, Róbert Kálmán: Der Maler Nagy Imre. A székely festö és az erdélyi szászok (1925-1935). Emil Witting regényrészleteivel. Csíkszereda: Csíkszereda 
Kiadóhivatal 2018 [Der Maler Nagy Imre. Der Szekler Maler und die Siebenbürger Sachsen (1925-1935). Emil Wittings Romanfragmente. Miercurea Ciuc/Szeklerburg: Verlag des Bürgermeisteramtes]. Mit zahlreichen Reproduktionen von Graphiken und Ölbildern des Künstlers.

Hönig, Alfred: Emil Witting. Der Karpatenjäger, der zum Dichter wurde. In: Südostdeutsche Vierteljahresblätter, 29. Jg., 1980, Folge 4, S. 271-275.

Kramer, Rainer: Findbuch zum Bestand Emil Witting (18801952). Archiv des Instituts für deutsche Kultur und Geschichte Südosteuropas (IKGS). München 2015, 17 S. Wagner, Udo Peter: Emil Witting. In: Die rumäniendeutsche Literatur in den Jahren 1918-1944. Redigiert von Wittstock, Joachim/Sienerth, Stefan. Bukarest: Kriterion Verlag 1992 (Beiträge zur Geschichte der rumäniendeutschen Dichtung), S. 217-225, 510f, Bildteil S. 14f. 